\title{
Teasing apart the effects of maths anxiety and test anxiety on arithmetic performance
}

\author{
Ngan Yin Chan ${ }^{1}$, Joey Tang ${ }^{2,3 *}$
}

\begin{abstract}
Previous studies have reported the relationship between high maths anxiety and poor maths achievement but the precise aspects of maths and the circumstances under which maths anxiety would have a negative effect on performance remained debatable. The present study set out to investigate the effects of maths anxiety on maths performance under both timed and untimed conditions using complex and simple arithmetical problems (with and without carry or borrow operation respectively). University students with high levels of maths anxiety performed significantly poorer than those with medium or low maths anxiety in complex but not simple addition and subtraction problems. This interaction between maths anxiety and problem complexity remained significant even when test anxiety levels were partialled out. There was no evidence to support time pressure as a source of anxiety. Our findings provide the first report of a detrimental effect of maths anxiety on arithmetic performance in adult participants, having controlled for test anxiety.
\end{abstract}

Keywords: mathematics anxiety, test anxiety, arithmetic performance

\section{INTRODUCTION}

The topic of maths anxiety has been investigated for decades and its definition has changed notably over the years. Despite increasing research in this area, there is no clear consensus as to what constitutes maths anxiety but researchers have generally accepted it as a condition characterised by negative reactions (e.g. fear) towards mathematical tasks $(1,2)$. According to the tripartite framework of emotion (3), maths anxiety manifests itself at the physiological, cognitive, as well as behavioural level, affecting individuals' heart and respiration rates (4), belief systems and emotions ( 1 ), as well as their study choices and career paths (5) respectively.

Numerous studies have reported the relationship between high maths anxiety and poor maths achievement (see (6) for a review), observed in secondary school students (7) and college students (8), particularly among those who perceived themselves as low achievers (9). Similarly, studies on pre-college students also indicated a negative correlation between maths anxiety and enjoyment of maths as well as self-rated confidence level in maths (10).

Previous research reported higher error rate and longer reaction time in highly maths anxious individuals when they were asked to verify "confusion" problems (e.g. $3+4=12$ where the result is the product of the two operands as opposed to the sum as suggested by the addition operation), while no difference was observed between individuals with different maths anxiety levels on simple arithmetic fact problems (e.g. $4+5=9$ ). Interestingly, highly maths anxious individuals were observed to sacrifice accuracy for shorter reaction time. This avoidance tendency was interpreted as the individuals' desire to escape the situation with maths as quickly as possible (11). In addition, highly maths anxious individuals found complex problems such as addition with a carry operation particularly difficult to solve (11). When such problems were answered correctly, these individuals took three times longer than the lowly maths anxious participants. The poor performance of highly maths anxious participants in complex questions was termed "anxiety complexity effect" (11). Apart from the carry operation, problem complexity can also be operationalised by problem size. For example, one study reported that highly maths anxious participants performed worse in two-digit compared to single-digit problems (12).

${ }^{1}$ Department of Psychiatry, The Chinese University of Hong Kong, Hong Kong SAR, China. ${ }^{2}$ Center for Language and Brain, Shenzhen Institute of Neuroscience, Shenzhen, China. ${ }^{3}$ Myndful Learning Association, Hong Kong SAR, China.

*Corresponding author. Email: joey.tang@uclmail.net (J.T.)
Research suggests that working memory plays an important role in solving maths problems. For example, a memory load task (i.e. memorising six letters) produced a detrimental effect on arithmetic performance as maintaining the letters consumed some of the limited working memory resources, leaving fewer resources for solving the arithmetic problems (13). Moreover, highly maths anxious participants with greater working memory capacity outperformed those with weaker working memory capacity in maths tasks; the latter group also reported to have higher levels of maths anxiety (14). These findings support the important role of working memory in maths tasks.

The processing efficiency theory, proposed to explain general anxiety effects on performance (15), has been extended to explain the detrimental effect of maths anxiety on maths performance (16). According to this theory, intrusive thoughts and worries evoked by maths content divert the individual's attention away from the maths task at hand and compete with ongoing cognitive processes for limited working memory resources in highly maths anxious individuals, leading to poor maths performance. Complex arithmetic problems with high demand for working memory are particularly affected.

An alternative explanation in terms of the inhibition theory (17) has also been adopted to explain performance deficits in maths anxious individuals (18). Highly maths anxious individuals are believed to have a deficient inhibition mechanism in suppressing taskirrelevant thoughts which consume working memory resources. Research indicated that these individuals required more time and made more errors in reading paragraphs which contained maths words that were unrelated to the text (18). In other words, they failed to inhibit task-irrelevant maths content.

Anxiety reactions such as intrusive thoughts and worries experienced by maths anxious individuals appear to constitute a major factor affecting their maths performance. These anxiety reactions can be triggered first by the maths content itself and second by a time pressure (12). Worrying about the time constraint on a maths task competes for memory resources with performing the task itself (15). When time constraint was removed, performance difference in terms of accuracy observed between highly and lowly maths anxious individuals on arithmetic problems was indeed reduced to minimal (12), providing support for the detrimental effect of time pressure on maths performance. However, inconsistent findings have been reported in this area. For example, although students 
made more errors in the timed condition, performance was similar across high- and low-maths anxiety groups (19), challenging the notion of time pressure as a key source of anxiety.

It is worth noting that majority of the previous studies did not typically control for test anxiety - a construct that correlates significantly with maths anxiety $(20,21)$. Some argued that maths anxiety may simply represent a form of test anxiety (1). To complicate matters further, tests (including maths tests) are usually done under timed conditions, so the reported detrimental effect of maths anxiety on arithmetic performance may again reflect, at least partially, a mere test anxiety effect.

The present study aimed to re-address the effect of maths anxiety on arithmetic performance under timed and untimed conditions, while controlling for individuals' test anxiety levels. Here, we included subtraction as well as addition problems; only the latter had previously been investigated. We anticipated a significant maths anxiety effect on arithmetic performance in complex but not simple arithmetic (i.e. addition with carry operation and subtraction with borrow operation compared to addition without carrying and subtraction without borrowing respectively), as the former demanded more working memory resources. We further investigated if this effect would occur only under timed condition. The majority of existing studies on maths anxiety recruited school-aged participants (e.g. $(9,22,23))$. However, maths anxiety is known to persist into adulthood and mild to moderate levels of maths anxiety are commonly reported in the general adult population (24). Thus, we investigated the effect of maths anxiety in a sample of young adults.

\section{RESULTS}

\section{Phase I}

Data from a sample of 199 university students revealed a significant correlation between maths anxiety (measured by the Mathematics Anxiety Rating Scale (MARS) (25)) and test anxiety (measured by the Test Anxiety Inventory (TAI) $(26))(\mathrm{r}=.451, p<.01)$. The mean scores of MARS and TAI were $66.5 \pm 9.3$ and $41.5 \pm 8.8$ respectively. The correlations between these measures were very similar for the male and female samples (both $p$ s $<.05$ ).

\section{Phase II}

Participants from Phase I were classified into three groups (low, medium and high maths anxiety groups), based on their MARS scores and re-invited to take part in Phase II. A total of 36 students were recruited including 11 in the low maths anxiety group (mean $=37, \mathrm{SD}=3.61), 13$ in the medium maths anxiety group (mean = $65, \mathrm{SD}=2.44)$ and 12 in the high maths anxiety group $($ mean $=98$, $\mathrm{SD}=3.87)$.

Error rates in the arithmetic tests were analysed using a 2 x 2 × 2 × 3 mixed-design ANOVA, with time pressure (timed or untimed), arithmetic operation (addition or subtraction), and problem complexity (simple or complex) as within-participants factors, and maths anxiety (MA) group (low MA, medium MA, or high MA) as the between-participants factor. A $p$ value of < .05 was considered statistically significant. All data analyses were performed using IBM SPSS Statistics.

The ANOVA revealed a significant main effect of time pressure $(\mathrm{F}(1,33)=4.40, p<.05)$. Participants made significantly more errors during timed test compared to untimed test. Mean error rate for subtraction problems was higher than that for addition problems but the difference failed to reach statistical significance $(\mathrm{F}(1,33)=3.60$, n.s. $)$. On the other hand, the main effect of problem complexity was significant $(\mathrm{F}(1,33)=38.84, p<.05)$. Significantly more errors were made when calculating complex compared to simple problems. The ANOVA revealed also a significant main effect of group $(\mathrm{F}(2,33)=4.40, p<.05)$. This factor showed a significant interaction only with problem complexity $(\mathrm{F}(2,33)=$ $3.64, p<.05$ ). All other interactions were non-significant (all n.s.).

A one-way ANOVA was conducted for each level of complexity. For simple problems, the main effect of group was non-significant $(\mathrm{F}(2,33)<1, n . s$. $)$. In contrast, there was a significant main effect of group when complex problems were considered $(\mathrm{F}(2,33)=5.50, p$ $<.05)$. Tukey's HSD post-hoc tests revealed significant differences between high and medium MA groups $(p<.05)$ and between high and low MA groups $(p<.05)$; the difference between medium and low MA groups was non-significant (n.s.). The mean error rates of $4.66 \%, 6.29 \%$ and $11.6 \%$ for low, medium and high MA group respectively (see Fig. 1).

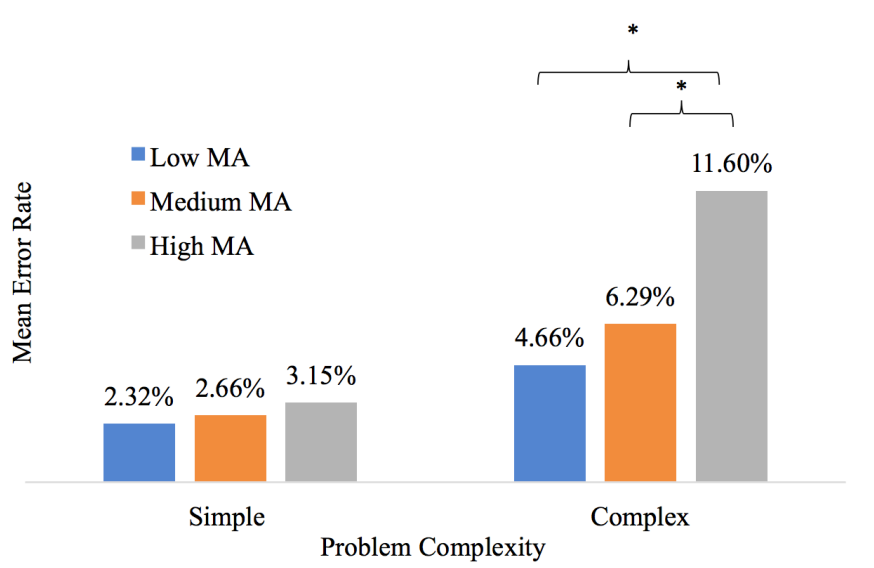

Fig. 1. Mean error rates as a function of problem complexity in different maths anxiety groups. $\mathrm{MA}=$ maths anxiety. ${ }^{*} p<.05$.

Given the significant correlation between maths anxiety (MARS) and test anxiety (TAI) scores observed in Phase I, we ran the ANOVA again incorporating test anxiety scores as the covariate. In this analysis, none of the within-participants main effects were significant (all n.s.). The covariate also did not yield a significant effect $(\mathrm{F}(1,32)<1$, n.s. $)$. There was, however, a significant main effect of group $(\mathrm{F}(2,32)=4.52, p<.05)$. More importantly, the group $\mathrm{x}$ complexity interaction remained significant $(\mathrm{F}(2,32)=$ $3.33, p<.05$ ). All other interactions were non-significant (all n.s.).

\section{DISCUSSION}

The present study re-examined the effect of maths anxiety on arithmetic performance under timed and untimed conditions, while controlling for individuals' test anxiety levels. Consistent with previous findings, time pressure has a negative effect on arithmetic performance but this effect is similar across individuals with different levels of maths anxiety (19). Our data also confirmed that highly maths anxious individuals' poor arithmetic performance is limited to complex but not simple problems $(11,12)$. Furthermore, this effect is observed not only in addition problems (with a carry operation) as previously reported but also in subtraction problems (with a borrow operation). The inflated error rate observed with complex problems in highly maths individuals may be explained in terms of the processing efficiency theory (15). Specifically, the high cognitive demand of complex problems competes with diverted attention from anxiety reactions for limited working memory resources, leading to the detrimental effect. Anxiety reactions such 
as intrusive and worrying thoughts act like a secondary task, adding load to the already limited working memory in highly maths anxious individuals (16). Thus, when they have to solve complex problems involving carrying or borrowing which rely on the use of working memory to keep track of calculation procedures, their performance suffers. It is also possible that highly maths anxious individuals have a defective inhibition mechanism and have difficulties ignoring distractors - in this case, intrusive thoughts and worries arisen from maths content - consuming working memory resources (18)

The fact that time pressure did not produce differential effects on arithmetic performance across individuals with different levels of maths anxiety suggests that it is an unlikely candidate for the source of anxiety. Time pressure may, on the other hand, be more associated with test anxiety as it is a feature typically found across different types of test. Although our data demonstrated a significant moderate correlation between test anxiety and maths anxiety, the detrimental effect of maths anxiety on arithmetic performance remained prominent even after partially out the effect of test anxiety, supporting the notion that maths anxiety and test anxiety are separate constructs, consistent with findings from a previous study using confirmatory factor analysis (21). Our findings highlight the unique contribution of maths anxiety to poor arithmetic performance.

Several caveats are worth mentioning when interpreting the current findings. Firstly, measurements of maths and test anxiety were based on self-reported inventories. A more objective approach such as monitoring participants' physiological arousal levels (e.g. heart and respiration rates, pupil dilation and galvanic skin response) during maths tasks could more accurately reflect individuals' anxiety levels. Secondly, the participation rate in Phase II was modest compared Phase I, with unknown reasons of refusal. It was possible that individuals with the highest levels of maths anxiety were more reluctant to take part in the maths problems in Phase II, biasing the sample. Thirdly, the generalisability of the current study is limited as we focused only on university students. Future research should include more mature adults, particularly those who are at work, to explore potential effects of maths anxiety in different work settings.

In conclusion, the present study provides insights into the unique effect of maths anxiety on arithmetic performance, when test anxiety has been partialled out. Our results confirm the notion that the importance of maths anxiety does not end when one leaves school (27). Young adults who participated in the current study were recruited from a broad range of majors (e.g. sociology, psychology, engineering, etc.) across different universities and they will enter into different careers which undoubtedly require maths skills (albeit with different levels of engagement). As maths anxiety is commonly reported in the general adult population, it is important to have a more comprehensive understanding of the nature of maths anxiety. We speculate the source of anxiety resides in the negative feelings associated with the motion of maths. However, more research is needed to pinpoint the exact aspects of maths (aside from problem complexity) that give rise to anxiety, so that effective intervention can be developed.

\section{MATERIALS AND METHODS}

\section{Study design}

The present study consisted of two phases. Phase I explored the distribution of maths anxiety among university students, while
Phase II examined the effect of maths anxiety on arithmetic performance. All young adults aged above 18 years studying at local universities were eligible to participate in the study. In Phase I, university students were invited to complete a set of questionnaires covering demographic information and statements to evaluate their maths and test anxiety levels. Participants were classified into subgroups according to their maths anxiety scores and re-invited to take part in Phase II which included two arithmetic tests. Ethical approvals were obtained from the university ethics committee prior to the commencement of data collection. Informed consent was obtained from the participating students.

\section{Phase I}

\section{Participants}

A total of 199 university students participated in Phase I (mean age $=21.04$ years, range $=18$ to 30 ), among who were 119 females $(59.8 \%)$. Participants were recruited from different colleges studying a broad range of majors, e.g. sociology, psychology, engineering, etc.

\section{Materials}

The 30-item Mathematics Anxiety Rating Scale (MARS) (25) is an inventory used to assess an individual's level of maths anxiety. A few of the MARS items were modified to accommodate for cultural differences. Participants were asked to rate how anxious they would be in both academic (e.g. taking the final maths examination) and everyday (e.g. reading a cash register receipt) situations. They rated each item on a 5-point scale, from "not at all" to "very much". A high level of maths anxiety is indicated by a high total MARS score. The 30-item MARS has been demonstrated to have a high test-retest reliability $(\mathrm{r}=.90)$ and a strong correlation $(\mathrm{r}=.92)$ with the 98 -item MARS (25).

The Test Anxiety Inventory (TAI) (26) is a 20-item measure used to assess an individual's anxiety related to test situations. It contains self-descriptive statements (e.g. I feel very jittery when taking an important test) which the participants rated on a 4-point scale to indicate the intensity of their feelings during those situations. The TAI has been demonstrated with high test-retest reliability ( $\mathrm{r}=$ .80) (28) and validity (29).

\section{Phase II}

\section{Participants}

Participants from Phase I were classified into three groups based on their MARS scores: 30-41 (below 10th percentile) for low maths anxiety, 63-68 (45th to 55th percentile) for medium maths anxiety and 94-123 (above 90th percentile) for high maths anxiety. This cutoff scheme was adopted to ensure the demarcation of participants would produce a true difference in their performance (10). These subgroups of participants $(n=61)$ were re-invited to participate in Phase II. A total of 36 students agreed to take part (mean age $=21.3$ years, range $=18$ to 26 ), among who were 19 females and 17 males.

\section{Procedures}

Participants were asked to perform two arithmetic tests under both timed and untimed conditions one week apart. The order of the tests was counterbalanced across participants. Under the timed condition, the amount of time allocated to each section was determined by a pilot study $(n=6)$ in which students were asked to complete the test as quickly and as accurately as they could. The time to complete each of the two sections was recorded separately. The time limit used in each section of the present study was set at 
$80 \%$ of the mean response time recorded in the pilot test. Prior to the timed test, participants were instructed to work as quickly and as accurately as possible within the time limit. Under the untimed condition, no time limit was set. Participants were allowed to take as much time as they needed to complete the test.

\section{Materials}

Participants were required to complete two 8-page arithmetic tests, one under timed and another under untimed condition. Each test contained eight sections of problems, namely, 1) simple two-digit addition (e.g. $37+52=$ ?), 2) two-digit addition with carrying (e.g. $26+58=$ ?), 3) simple two-digit subtraction (e.g. $79-53=$ ?), 4) two-digit subtraction with borrowing (e.g. $86-47=$ ?), 5) simple three-digit addition (e.g. $365+232=$ ?), 6) three-digit addition with carrying (e.g. $345+627=$ ?), 7) simple three-digit subtraction (e.g. $796-324=$ ?), 8) three-digit subtraction with borrowing (e.g. 571 $-248=$ ?).

All the numbers used in the tests were composed of digits from 1 to 9 . Numbers were excluded with the following criteria: a) operands with digit 0 (e.g. 20) to avoid the use of rule-based solutions (30), b) ties numbers (e.g. 66) to avoid the "tie effect" (31, $32)$, c) operand pairs with answer of 5 or 0 in the unit place to avoid the "five effect" (33). The frequency of each digit appearing in the unit or decade column for two-digit problems was balanced in each section to avoid practice effect. For three-digit addition problems with carrying and subtraction problems with borrowing, the frequency of the carry or borrow operation occurring at the unit or decade column was also balanced.

In the timed condition, error rate was calculated by the number of incorrect answers divided by the number of problems attempted (19). For example, if a participant attempted 14 out of 20 problems and made 2 errors, the error rate would be $14.29 \%$. However, if the participant did not complete the last problem he/she was attempting, it would not be considered an error because the incompletion was due to the time constraint but not to his/her lack of competence. For example, if the participant answered " 8 " to the problem " $45+23=$ ?" which was the last question that he/she attempted, then it would not be considered an error. In the untimed condition, however, any problems that were left unanswered would be considered errors as participants were given unlimited time to complete the test.

\section{REFERENCES}

1. F. C. Richardson, R. L. Woolfolk, Test Anxiety: Theory, Research, and Application, I. G. Sarason, ed. (Hillsdale, NJ: Erlbaum, 1980), pp. 271-288.

2. M. H. Ashcraft, Math anxiety: Personal, educational, and cognitive consequences. Curr. Dir Psychol. Sci. 11, 181-185 (2002).

3. P. J. Lang, Research in Psychotherapy, J. M. Schlien, ed. (American Psychological Association, Washington, DC, 1968), vol. III.

4. M. W. Faust, H. J. Johnson, M. H. Ashcraft, Analysis physiological reactivity in mathematics phobia. (Unpublished manuscript, 1996).
5. S. F. Chipman, D. H. Krantz, R. Silver, Mathematics anxiety and science careers among able college women. Psychol. Sci. 3, 292-296 (1992).

6. R. Hembree, The nature, effects, and relief of mathematics anxiety. J. Res. Math. Educ. 21, 33-46 (1990).

7. J. L. Meece, A. Wigfield, J. S. Eccles, Predictors of math anxiety and its influence on young adolescents' course enrollment intentions and performance in mathematics. J. Educ. Psychol. 82, 60-70 (1990)

8. E. Fennema, J. Sherman, Sex-related differences in mathematics achievement, spatial visualization and affective factors. Am. Educ. Res. J. 14, 51-71 (1977).

9. M. A. Al Mutawah, The influence of mathematics anxiety in middle and high school students math achievement. Int. Educ. 8, 239-252 (2015).

10. M. H. Ashcraft, K. S. Ridley, Handbook of Mathematical Cognition, J. I. D. Campbell, ed. (Psychology Press, New York, 2005), pp. 315-327.

11. M. H. Ashcraft, M. W. Faust, Mathematics anxiety and mental arithmetic performance: An exploratory investigation. Cogn. Emot. 8, 97-125 (1994).

12. M. W. Faust, M. H. Ashcraft, D. E. Fleck, Mathematics anxiety effects in simple and complex addition. Mathematical Cognition 2, 25-62 (1996).

13. M. H. Ashcraft, E. P. Kirk, The relationships among working memory, math anxiety, and performance. J. Exp. Psychol. Gen. 130, 224-237 (2001).

14. H. Miller, J. Bichsel, Anxiety, working memory, gender, and math performance. Pers. Individ. Differ. 37, 591-606 (2004).

15. M. W. Eysenck, M. G. Calvo, Anxiety and performance: The processing efficiency theory. Cogn. Emot. 6, 409-434 (1992).

16. M. H. Ashcraft, Cognitive psychology and simple arithmetic: A review and summary of new directions. Mathematical Cognition 1, 3-34 (1995).

17. L. Hasher, R. T. Zacks, Working memory, comprehension, and aging: A review and a new view. Psychol. Learn. Motiv. 22, 193-225 (1988).

18. D. R. Hopko, M. H. Ashcraft, J. Gute, Mathematics anxiety and working memory: Support for the existence of a deficient inhibition mechanism. J. Anxiety Disord. 12, 343-355 (1998).

19. J. S. Kellogg, D. R. Hopko, M. H. Ashcraft, The effects of time pressure on arithmetic performance. J. Anxiety Disord. 13, 591-600 (1999).

20. L. H. Chiu, L. L. Henry, Development and validation of the mathematics anxiety scale for children. Meas. Eval. Couns. Dev. 23, 121-127 (1990).

21. R. Kazelskis, C. Reeves, M. E. Kersh, G. Bailey, K. Cole, M. Larmon, L. Hall, D. C. Holliday, Mathematics anxiety and test anxiety: Separate constructs? J. Exp. Educ. 68, 137-146 (2000)

22. A. Devine, K. Fawcett, D. Szücs, A. Dowker, Gender differences in mathematics anxiety and the relation to mathematics performance while controlling for test anxiety. Behav. Brain Funct. 8, 33 (2012).

23. M. C. Passolunghi, C. D. Vita, S. Pellizzoni, Math anxiety and math achievement: The effects of emotional and math strategy training. Dev. Sci. pp. e12964 (2020).

24. S. A. Hart, C. M. Ganley, The nature of math anxiety in adults: Prevalence and correlates. J. Numer. Cogn. 5, 122-139 (2019)

25. R. M. Suinn, E. H. Winston, The mathematics anxiety rating scale, a brief version: Psychometric data. Psychol. Rep. 92, 167-173 (2003).

26. C. D. Spielberger, Test Anxiety Inventory: Preliminary Professional Manual. (Consulting Psychologists Press, Palo Alto, CA, 1980).

27. K. Skagerlund, T. Lind, C. Strömbäck, G. Tinghög, D. Västtjäll, Financial literacy and the role of numeracy-How individuals' attitude and affinity with numbers influence financial literacy. $J$. Behav. Exp. Econ. 74, 18-25 (2018).

28. C. D. Spielberger, H. P. Gonzalez, C. J. Taylor, B. Algaze, W. D. Anton, Stress and Anxiety, C. D. Spielberger, I. G. Sarason, eds. (Wiley, New York, 1978), vol. 5, pp. 167-191.

29. I. G. Sarason, Stress and Anxiety, C. D. Spielberger, I. G. Sarason, eds. (Wiley, New York, 1978), vol. 5, pp. 193-216.

30. M. H. Ashcraft, Cognitive arithmetic: A review of data and theory. Cognition 44, 75-106 (1992).

31. B. Butterworth, M. Zorzi, L. Girelli, A. R. Jonckheere, Storage and retrieval of addition facts: The role of number comparison. Q. J. Exp. Psychol. 54, 1005-1029 (2001).

32. J. I. Campbell, R. Gunter, Calculation, culture, and the repeated operand effect. Cognition $\mathbf{8 6}$, 71-96 (2002).

33. R. S. Siegler, Strategy choice procedures and the development of multiplication skill. J. Exp. Psychol. Gen. 117, 258-275 (1988).

Acknowledgements: Funding: This study was self-funded. Author contributions: Dr. Ngan Yin Chan and Dr. Joey Tang contributed to the experimental design, data interpretation and preparation of the manuscript. In addition, Dr. Chan contributed to data collection and analysis. Competing interests: The authors declare no competing interests, financial or otherwise, in completion of this work. Data and materials availability: All data and materials are available are upon request to the corresponding author. 